\title{
HARMONIC ANALYSIS AND BOUNDARY CORRESPONDENCE UNDER QUASICONFORMAL MAPPINGS
}

\author{
Jan G. Krzyż
}

\section{Introduction. Notations. Statement of results}

Any quasiconformal (abbreviated: qc) self-mapping of a Jordan domain $G$ has a homeomorphic extension to its closure $\bar{G}$ and induces this way a sense-preserving homeomorphism of the boundary curve $\Gamma$ onto itself. In other words, any qc automorphism of $G$ induces an automorphism of $\partial G$. It is well-known that the induced automorphisms of $\Gamma$ with a fixed point can be represented, after a suitable conformal mapping of $G$ onto $U:=\{w: \operatorname{Im} w>0\}$, by $M$-quasisymmetric (abbreviated: $M$-qs) functions, i.e. functions $h: \mathbf{R} \rightarrow \mathbf{R}$ which satisfy for some $M \geq 1$ the celebrated $M$-condition of Beurling-Ahlfors: There exists a constant $M \geq 1$ such that

$$
M^{-1} \leq \frac{h(x+t)-h(x)}{h(x)-h(x-t)} \leq M ; \quad x \in \mathbf{R}, 0 \neq t \in \mathbf{R}
$$

cf. [1], [2].

As pointed out by the author in an earlier paper [5], the induced automorphisms of $\Gamma$ can be also characterized by $M$-qs functions of the form $x \rightarrow x+\sigma(x)$, where $\sigma$ is periodic with period $2 \pi$. In fact, any automorphism of $\Gamma$ admitting a qc extension $H$ to $G$ also admits a qc extension $\tilde{L} \circ H$ to $G$ having a fixed point $z_{0} \in G$, where $\tilde{L}$ is a qc automorphism of $G$ (with complex dilatation of a constant absolute value) that keeps the points of $\Gamma$ fixed and carries $H\left(z_{0}\right)$ to $z_{0}$. The automorphism $\tilde{L}$ of $G$ corresponds, after a conformal mapping $g$ of $G$ onto the upper halfplane $U$, to the affine automorphism $L$ of $U$ :

$$
w \mapsto L(w)=L\left(w ; w_{1}, w_{2}\right)=\left(w_{1}-\bar{w}_{1}\right)^{-1}\left(\left(w_{2}-\bar{w}_{1}\right) w+\left(w_{1}-w_{2}\right) \bar{w}\right),
$$

where $w_{1}=g \circ H\left(z_{0}\right), w_{2}=g\left(z_{0}\right)$. Evidently $L\left(w_{1}\right)=w_{2}$ and $L(x)=x$ for any $x \in \mathbf{R}$. If $\tilde{L}=g^{-1} \circ L \circ g$, then $\tilde{L} \circ H$ and $H$ are qc automorphisms of $G$ inducing the same automorphism of $\partial G$, while $\tilde{L} \circ H\left(z_{0}\right)=z_{0}$. Now, the qc automorphism $\tilde{L} \circ H$ composed first with a conformal mapping of $G$ onto the unit disk $\mathbf{D}$ with $z_{0}$ corresponding to the origin, and next with the conformal mapping $z \mapsto-i \log z$ of 
$\mathbf{D} \backslash\{0\}$ onto $U$, generates a qc automorphism of $U$. This automorphism induces a quasisymmetric function of the desired form $x \mapsto x+\sigma(x)$ from which the original automorphism of $\Gamma$ can be recovered.

The above mentioned representation of the boundary correspondence under qc mappings has many advantages. Due to periodicity of $\sigma$, some local properties, e.g. local Hölder continuity, become global ones. Moreover, the exceptional rôle of the point at infinity, which is not justified while dealing with the boundary correspondence, vanishes in this approach. The functions $\sigma$, which may be considered as deviations of the induced automorphisms from the identity, have many properties that are very nice from the point of view of the classical harmonic analysis. They are of monotonic type (i.e. $\sigma(x)+C x$ is strictly monotonic for some $C \neq 0$ ), of bounded variation over $[0 ; 2 \pi]$ and belong to the familiar class $\Lambda_{\alpha}$ with $\alpha$ defined by (1.7).

Obviously the period $2 \pi$ may be replaced by an arbitrary $a>0$. The class of all periodic functions $\sigma$ of period $a>0$ such that $x \mapsto x+\sigma(x)$ is $M$-qs on $\mathbf{R}$ will be denoted by $E(M, a)$. By performing a suitable translation we can normalize $\sigma \in E(M, a)$ so that

$$
\sigma(0)=0=\sigma(a)
$$

the subclass of $E(M, a)$ with the normalization $(0.2)$ will be denoted by $E_{1}(M, a)$. Evidently $E_{1}(M, 1)+$ id is the subclass of the familiar class $H_{1}(M)$ of $M$-qs functions $h$ normalized by the condition

$$
h(0)=0, \quad h(1)=1 .
$$

With any $\sigma \in E(M, a)$ we may also associate the function

which satisfies

$$
\sigma_{0}(x)=\sigma(x)-a^{-1} \int_{0}^{a} \sigma(t) d t
$$

$$
\int_{0}^{a} \sigma_{0}(x) d x=0
$$

For various reasons this normalization seems to be most natural and the resulting subclass $E_{0}(M, a)$, or rather the classes $E_{0}(M, 1), E_{0}(M, 2 \pi)$ will be the main objects of our investigations. In the latter case we adopt an abbreviated notation $E(M)$. If (0.4) holds then $\sigma_{0}\left(x_{0}\right)=0$ for some $x_{0} \in[0 ; a)$ so that $\sigma_{1}(t)=$ $\sigma_{0}\left(t+x_{0}\right)$ belongs to $E_{1}(M, a)$. Thus both classes correspond to each other under a translation. Obviously, $\sigma \in E_{k}(M, a), k=0,1$ if and only if a suitable normalization (0.2), or (0.4), holds and $x \mapsto x+\sigma(x)$ is $M$-qs, i.e. $\sigma$ satisfies

$$
M^{-1} \leq \frac{t+\sigma(x+t)-\sigma(x)}{t+\sigma(x)-\sigma(x-t)} \leq M ; \quad x \in \mathbf{R}, 0 \neq t \in \mathbf{R} .
$$

Remark 0.1. As shown in [5], inequality (0.5) with the same $M$ holds if and only if $x$ ranges over $[0 ; a]$ and $0<t<\frac{1}{2} a$. 
While establishing general properties of $\sigma$, we shall be mainly concerned with the class $E_{1}(M, 1)$. On the other hand, we prefer to take $a=2 \pi$ while dealing with Fourier series of $\sigma$. In Section 1 some obvious consequences of the definition are obtained. E.g. the modulus of continuity of $\sigma \in E(M, 1)$ is determined and an analytic expression for a qc extension of $x+\sigma(x)$ in terms of Fourier coefficients of $\sigma$ is given. In Section 2 an estimate of the sup-norm of $\sigma+E_{1}(M, 1)$ is obtained. Also an estimate of $\int_{0}^{1}|\sigma|$ is derived. The Fourier series of $\sigma(x)$ can be written in the form $\sum_{n=1}^{\infty} \varrho_{n} \sin \left(n x+x_{n}\right), \varrho_{n} \geq 0$, and then the norm estimates of $\sigma$ enable us to find finite bounds for $\sum \varrho_{n}$ and $\sum n \varrho_{n}^{2}$ in $E(M)$. In Section 3 the existence of the uniform bound $c(M)$ for the sequence $\left(n \varrho_{n}\right)$ in $E(M)$ is established and two-sided estimates of $c(M)$ are given. The problem of Fourier coefficients of $\sigma$ is reduced to the determination of the constant $c(M)=\sup \left\{\varrho_{1}(\sigma): \sigma \in E(M)\right\}$.

The results presented in this paper were announced at the $13^{\text {th }}$ Rolf Nevanlinna Colloquium in Joensuu on August 13, 1987 [cf. p. 32 of Abstracts of lectures presented at the Colloquium]. The author would like to express his sincere thanks to the referee for his very helpful criticism.

\section{Some preliminary considerations and remarks}

If $a>0$ and $k=0,1$ then

$$
\sigma(x) \in E_{k}(M, a) \quad \Leftrightarrow \quad a^{-1} \sigma(a x) \in E_{k}(M, 1) .
$$

Thus taking $a=1$ does not impair generality. Similarly, if $\sigma$ has the period $a>0$ and $n \in \mathbf{N}$, then

$$
\sigma(n x) \in E_{0}(M, a) \quad \Leftrightarrow \quad n \sigma(x) \in E_{0}(M, a) .
$$

In fact, the middle term for $\sigma(n x)$ in (0.5) has the form

$$
\frac{t+\sigma(n x+n t)-\sigma(n x)}{t+\sigma(n x)-\sigma(n x-n t)}=\frac{n t++n \sigma(n x+n t)-n \sigma(n x)}{n t+n \sigma(n x)-n \sigma(n x-n t)}
$$

and the last expression may be considered as the middle term for $n \sigma(x)$. Furthermore, the normalization (0.4) is preserved.

The inequality (0.5) may be written in an equivalent form:

$$
M^{-1}(t+\sigma(x)-\sigma(x-t)) \leq t+\sigma(x+t)-\sigma(x) \leq M(t+\sigma(x)-\sigma(x-t)) .
$$

If $k=0,1$ is fixed, $\lambda_{j} \in[0 ; 1], \sigma_{j} \in E_{k}(M, a)$ for $j=1,2, \ldots, n$ and $\sum_{j=1}^{n} \lambda_{j}=1$, then

$$
\sum_{j=1}^{n} \lambda_{j} \sigma_{j} \in E_{k}(M, a)
$$

which is readily obtained from (1.3). 
Corollary 1.1. $E_{k}(M, a), k=0,1$, are convex subsets of the linear space $C(\mathbf{R})$ of continuous, real-valued functions on $\mathbf{R}$.

If $h \in H_{1}(M)$, so does $1-h(1-x)$. Consequently

$$
\sigma \in E_{k}(M, 1) \quad \Leftrightarrow \quad-\sigma(-x) \in E_{k}(M, 1) \quad k=0,1 .
$$

Corollary 1.2. If $\sigma \in E_{0}(M, 1)$, so does its odd part $\frac{1}{2}[\sigma(x)-\sigma(-x)]$. However, an analogous statement does not hold for the even part of $\sigma$.

The well-known local Hölder continuity of $M$-qs functions becomes a global property in $E(M, 1)$. We have

Proposition 1.3. If $h(x)=x+\sigma(x)$ and $\sigma \in E(M, 1)$ then the moduli of continuity $\omega(\delta ; f)$ satisfy for $0<\delta<\frac{1}{2}$ the inequality

$$
\omega(\delta ; f) \leq(2 \delta)^{\alpha}=\left(1+M^{-1}\right) \delta^{\alpha} \quad \text { with } \quad f=h, \sigma,
$$

where

$$
\alpha=\log _{2}\left(1+M^{-1}\right)
$$

Proof. With $f=h$ the inequality (1.6) is essentially due to Kelingos [4], also cf. [1], p. 66. Hence with $f=\sigma \in E_{1}(M, 1)$ we have $h(x)=x+\sigma(x) \in H_{1}(M)$ and

$$
h(t+x)-h(t)<(h(t+1)-h(t))(2 \delta)^{\alpha}=(2 \delta)^{\alpha}
$$

for any $t \in \mathbf{R}$ and $0<x<\delta<\frac{1}{2}$. Consequently,

$$
(2 \delta)^{\alpha}>h(t+x)-h(t)=x+\sigma(t+x)-\sigma(t)>0,
$$

or

$$
(2 \delta)^{\alpha}>\sigma(t+x)-\sigma(t)>-x>-\delta
$$

which implies (1.6) for $f=\sigma$.

Remark 1.4. $E_{k}(M, 1), k=0,1$, are convex, compact subsets of the space $C(\mathbf{R})$.

For convexity cf. Corollary 1.1. The compactness of $E_{1}(M, 1)$ follows from the Ascoli-Arzela theorem. The same is true for $E_{0}(M, 1)$. 
Corollary 1.5. If $\sigma \in E(M)$, then for any $t \in \mathbf{R}$

$$
|\sigma(t+x)-\sigma(t)|<2 \pi(\delta / \pi)^{\alpha}, \quad 0<x<\delta<\pi .
$$

Corollary 1.6. The class $E(M)$ is a subclass of the familiar class $\Lambda_{\alpha}$ of $2 \pi$-periodic, Hölder continuous functions with the exponent $\alpha$ given by (1.7). Thus the Fourier series of $\sigma$ and its conjugate $\tilde{\sigma}$ are absolutely and uniformly convergent on $\mathbf{R}$, cf. [6].

We shall now perform the Beurling-Ahlfors construction of the qc extension of an $M$-qs function $x \mapsto x+\sigma(x)$. Suppose that $\sigma \in E(M)$. Then we have for suitably chosen $x_{n} \in \mathbf{R}$

$$
\sigma(x)=\sum_{n=1}^{\infty} \varrho_{n} \sin \left(n x+x_{n}\right), \quad \varrho_{n} \geq 0 .
$$

The qc extension to the upper half-plane, cf. [1], takes the form $w=u(x, y)+$ $i v(x, y)$, where

$$
\begin{gathered}
u(x, y)=x+\sum_{n=1}^{\infty} \varrho_{n} \sin \left(n x+x_{n}\right) \sin n y /(n y) \\
v(x, y)=\frac{1}{2} y+\sum_{n=1}^{\infty} \varrho_{n} \cos \left(n x+x_{n}\right)(1-\cos n y) /(n y) .
\end{gathered}
$$

We see that $u, v$ are obtained by linear transformations generated by convergencepreserving factors, as applied to a convergent Fourier series (1.11) and its conjugate. The factors in (1.12) are even sum-preserving as $y \rightarrow 0$ and correspond to the Lebesgue method of summability, cf. [6]. On the other hand, the convergencepreserving factors in (1.13) tend to 0 as $y \rightarrow 0$ and this implies $\lim _{y \rightarrow 0} v(x, y)=0$ for any $x \in \mathbf{R}$. Note that $\left(\lambda_{n}\right)$ are convergence-preserving factors, if and only if the series $\sum\left|\lambda_{n}-\lambda_{n+1}\right|$ is convergent, cf. e.g. [3, p. 394].

\section{Estimates of $\|\sigma\|$. Some applications}

In this section we shall be concerned with estimates of various norms of $\sigma \in$ $E(M, a), M>1$. We obtain estimates of the form $A(M-1) /(M+1)$ with a universal constant $A$ independent of $M$ for the sup-norm and $L^{p}$-norm, $p=1,2$. We first prove 
Theorem 2.1. If $h \in H_{1}(M)$ then

$$
h(x) \leq x+(M-1) /(M+1), \quad 0<x<1 .
$$

Proof. It is sufficient to prove (2.1) with $h$ replaced by some function $F$ majorizing all $h \in H_{1}(M)$ on $I=[0 ; 1]$. A majorant $F$ can be obtained in a familiar way as the limiting function of the sequence $\left(L_{n}\right)$ of continuous, piecewise linear functions, defined as follows.

Let $\Pi_{n}$ be the partition of $I$ determined by the points $x_{k}^{(n)}=k \cdot 2^{-n}, k=$ $0,1, \ldots, 2^{n}$. We assume that $L_{0}=$ id and $L_{n}$ are linear in $I_{k}^{(n)}=\left[x_{k-1}^{(n)} ; x_{k}^{(n)}\right]$. Given $L_{n}$ and its values $y_{k}^{(n)}=L_{n}\left(x_{k}^{(n)}\right)$ we assume that the values of $L_{n}$ and $L_{n+1}$ coincide at $x_{k}^{(n)}$, while at the new points $x_{k}^{\prime}=\frac{1}{2}\left[x_{k-1}^{(n)}+x_{k}^{(n)}\right]$ of the partition $\Pi_{n+1}$ the values $y_{k}^{\prime}=L_{n+1}\left(x_{k}^{\prime}\right)$ are the maximal values admissible under the $M$ condition as applied to the end points of $I_{k}^{(n)}$ and its centre, i.e.

$$
y_{k}^{\prime}=(M+1)^{-1}\left(y_{k-1}^{(n)}+M y_{k}^{(n)}\right) \text {. }
$$

Evidently $\left(L_{n}\right)$ is an increasing sequence converging uniformly to the continuous increasing function $F$ being a majorant of all $h \in H_{1}(M)$ on $I$. Note that all $L_{n}$ are Hölder continuous with the exponent $\alpha$. We shall now obtain an estimate for the increment of

$$
\gamma_{n}=\max \left\{L_{n}(x)-x: x \in I\right\} .
$$

Suppose that $\gamma_{n+1}=L_{n+1}\left(x_{j}^{(n)}\right)$ for some $j$. Since $\left(\gamma_{n}\right)$ is increasing and $L_{n+1}\left(x_{j}^{(n)}\right)=L_{n}\left(x_{j}^{(n)}\right)$, we have $\gamma_{n+1}=\gamma_{n}$ in this case. Thus we may assume that $\gamma_{n+1}=L_{n+1}\left(x^{\prime}\right)=y^{\prime}$, where $x^{\prime}$ is the centre of an interval $I_{j}^{(n)}=\left[x_{1} ; x_{2}\right]$. Putting $y_{k}=L_{n}\left(x_{k}\right), k=1,2$, we see that $y^{\prime}$ has the representation (2.2). If $m=\left(y_{2}-y_{1}\right) /\left(x_{2}-x_{1}\right)$ is the slope of the segment on the graph of $L_{n}$ corresponding to the interval $I_{j}^{(n)}$, then after the bisection of $I_{j}^{(n)}$ relevant slopes on the graph of $L_{n+1}$ will be equal

$$
m^{\prime}=2 m M /(M+1), \quad m^{\prime \prime}=2 m /(M+1) .
$$

If $m \geq \frac{1}{2}(M+1)$, or $m \leq(M+1) / 2 M$, then $L_{n+1}-$ id will change monotonically on $I_{j}^{(n)}$ which implies that its maximal value $\gamma_{n+1}$ is attained at $x_{1}$ or $x_{2}$ and consequently $\gamma_{n+1}=\gamma_{n}$, as before. Otherwise

$$
(M+1) / 2 M<m<(M+1) / 2 .
$$


If $1 \leq m<(M+1) / 2$, then $L_{n}-$ id increases on $I_{j}^{(n)}$ to the value $y_{2}-x_{2}$. With $d=x_{2}-x_{1}=2^{-n}$ we have $y_{2}-y^{\prime}=m^{\prime \prime}\left(x_{2}-x^{\prime}\right)$, or in view of $(2.4)$

$$
\begin{aligned}
0 & \leq \gamma_{n+1}-\gamma_{n} \leq y^{\prime}-x^{\prime}-\left(y_{2}-x_{2}\right)=\frac{1}{2} d\left(1-m^{\prime \prime}\right) \\
& =\frac{1}{2} d(M+1-2 m) /(M+1) \leq \frac{1}{2} d(M-1) /(M+1) .
\end{aligned}
$$

If $(M+1) / 2 M<m<1$, then $L_{n}-\mathrm{id}$ decreases strictly on $I_{j}^{(n)}$ and its maximum on this interval is equal $y_{1}-x_{1}$. Then we have

$$
\begin{aligned}
0 & \leq \gamma_{n+1}-\gamma_{n} \leq y^{\prime}-x^{\prime}-\left(y_{1}-x_{1}\right)=\frac{1}{2} d\left(m^{\prime}-1\right) \\
& =\frac{1}{2} d(2 m M /(M+1)-1)<\frac{1}{2} d(M-1) /(M+1) .
\end{aligned}
$$

Thus in any case

$$
0 \leq \gamma_{n+1}-\gamma_{n} \leq \frac{1}{2} d(M-1) /(M+1)=2^{-n-1}(M-1) /(M+1) .
$$

Obviously $\gamma_{1} \leq \frac{1}{2}(M-1) /(M+1)$ so that $(2.6)$ implies

$$
\gamma_{n} \leq\left(2^{-1}+2^{-2}+\cdots+2^{-n}\right)(M-1) /(M+1)<(M-1) /(M+1)
$$

and this ends the proof. is true.

Note that for $1 /(M+1)<x<1$ a better trivial estimate $h(x)<x+(1-x)$

Corollary 2.2. If $h \in H_{1}(M)$, then for any $x \in I$

$$
|h(x)-x| \leq(M-1) /(M+1) \text {. }
$$

In fact, if $h(x) \geq x$ for some $x \in I$, then (2.7) follows immediately from (2.1). If $h(x)<x$, apply $(2.1)$ to $h_{1}(x)=1-h(1-x)$.

Corollary 2.3. If $\sigma \in E_{k}(M, 1), k=0,1$, then

$$
\sup \{|\sigma(x)|: x \in \mathbf{R}\} \leq(M-1) /(M+1) .
$$

If $\sigma \in E(M, 1)$ and $M[\sigma]=\max \{\sigma(x): x \in \mathbf{R}\}, m[\sigma]=\min \{\sigma(x): x \in \mathbf{R}\}$, then

$$
M[\sigma]-m[\sigma] \leq(M-1) /(M+1) .
$$

Corollary 2.4. If $\sigma \in E(M)$, then

$$
\sup \{|\sigma(x)|: x \in \mathbf{R}\} \leq 2 \pi(M-1) /(M+1):=2 m(M) .
$$

The constant $m(M)$ will appear later on in various estimates concerning the class $E(M)$. 
Corollary 2.5. Any $\sigma \in E(M, a)$ is a function of bounded total variation at most $2 a$ on the interval of periodicity $[0 ; a]$. In particular, for $\sigma \in E(M)$ we have

$$
V[\sigma] \leq 4 \pi \text {. }
$$

Some natural open problems arise whether the bounds just mentioned could be improved. In particular, the inequality (2.9) suggests that the bound in (2.8) with $k=0$ could be possibly replaced by $\frac{1}{2}(M-1) /(M+1)$. One may ask whether the bound in (2.11) could be replaced by a smaller term depending on $M$.

We shall be now concerned with bounds of integrals of $\sigma$. We have

Theorem 2.6. If $\sigma \in E_{0}(M, 1)$, then

$$
\int_{0}^{1}|\sigma(x)| d x \leq \frac{1}{4}(M-1) /(M+1) .
$$

Proof. Suppose first that $h(x)=x+\sigma(x) \in H_{1}(M)$. Then $1 /(M+1) \leq$ $\int_{0}^{1} h(x) d x \leq M /(M+1)$, cf. [1, p. 67]. Hence

$$
\left|\int_{0}^{1}(h(x)-x) d x\right| \leq \frac{1}{2}(M-1) /(M+1) \text {. }
$$

Thus, if $h(x)-x$ has a constant sign in $(0 ; 1)$, then

$$
\int_{0}^{1}|h(x)-x| d x \leq \frac{1}{2}(M-1) /(M+1) .
$$

Suppose now that $h(x)-x$ vanishes at the end-points of an interval $I \subset[0 ; 1]$ and has a constant sign in $I$. Then, after a similarity transformation of ratio $|I|$, we reduce the problem to the just considered one and obtain

$$
\int_{I}|h(x)-x| \leq \frac{1}{2}|I|^{2}(M-1) /(M+1) .
$$

Let $I_{k}$ (or $J_{l}$, respectively) be the systems of maximal, disjoint, open intervals in $(0 ; 1)$ such that $h(x)-x>0$ on $I_{k}$ (and $h(x)-x<0$ on $J_{l}$, respectively) so that $h(x)-x=0$ at the end-points of $I_{k}$ and $J_{l}$. If $h(x)-x=\sigma(x) \in E_{0}(M, 1)$ and $\sigma(x) \not \equiv 0$, then both systems are non-void. Moreover, $\sum \int_{I_{k}} \sigma=\sum \int_{J_{l}}(-\sigma)$ due to the normalization (0.4). Hence

$$
\int_{[0: 1]}|\sigma|=2 \sum \int_{I_{k}} \sigma=2 \sum \int_{J_{l}}(-\sigma) .
$$


Since $\sum\left|I_{k}\right|+\sum\left|J_{l}\right| \leq 1$, at least one of these sums, say $\sum\left|I_{k}\right|$, is $\leq \frac{1}{2}$ so that $\sum\left|I_{k}\right|^{2} \leq \frac{1}{4}$. Hence, by (2.13)

$$
\int_{[0: 1]}|\sigma|=2 \sum \int_{I_{k}} \sigma \leq 2 \sum \frac{1}{2}\left|I_{k}\right|^{2} \frac{M-1}{M+1} \leq \frac{1}{4} \frac{M-1}{M+1} .
$$

Corollary 2.7. If $\sigma \in E(M)$, then with the notation (2.10)

$$
\int_{0}^{2 \pi}|\sigma(x)| d x \leq \pi m(M)
$$

Proposition 2.8. If $\sigma \in E_{0}(M, 1)$, then

$$
\int_{0}^{1}|\sigma(x)|^{2} d x \leq \frac{1}{4}\left(\frac{M-1}{M+1}\right)^{2}
$$

Proof. If $\sigma \in E_{0}(M, 1)$, then

$$
\int_{0}^{1}|\sigma(x)|^{2} d x \leq \max |\sigma(x)| \int_{0}^{1}|\sigma(x)| d x \leq \frac{1}{4}\left(\frac{M-1}{M+1}\right)^{2} .
$$

in view of (2.8) and (2.12).

Corollary 2.9. If $\sigma \in E(M)$, then

$$
\int_{0}^{2 \pi}|\sigma(x)|^{2} d x \leq 2 \pi(m(M))^{2} .
$$

The norm estimates just obtained give rise to some inequalities connected with Fourier series of $\sigma$. In view of Corollary $1.6 \sigma \in E(M)$ can be represented by an absolutely and uniformly convergent Fourier series

$$
\sigma(x)=\sum_{n=1}^{\infty}\left(a_{n} \cos n x+b_{n} \sin n x\right) .
$$

Putting

$$
\sin x_{n}=a_{n}\left(a_{n}^{2}+b_{n}^{2}\right)^{-1 / 2}, \quad \cos x_{n}=b_{n}\left(a_{n}^{2}+b_{n}^{2}\right)^{-1 / 2}
$$

we can rewrite (2.17) in the form

$$
\sigma(x)=\sum_{n=1}^{\infty} \varrho_{n} \sin \left(n x+x_{n}\right), \quad \varrho_{n}=\left(a_{n}^{2}+b_{n}^{2}\right)^{1 / 2} \geq 0 .
$$


The relation $E(M) \subset \Lambda_{\alpha} \cap V B[0 ; 2 \pi]$ supplies us with much information on the Fourier series of $\sigma$. E.g. for any $\beta<\alpha$

$$
\sum_{n=1}^{\infty} n^{\beta / 2}\left(\left|a_{n}\right|+\left|b_{n}\right|\right)<+\infty
$$

(cf. [6, p. 251]. Moreover,

$$
\sum_{n=1}^{\infty}\left(\left|a_{n}\right|^{\gamma}+\left|b_{n}\right|^{\gamma}\right)<+\infty, \quad \sum_{n=1}^{\infty}\left(a_{n}^{2}+b_{n}^{2}\right)^{\gamma / 2}<+\infty
$$

for any $\gamma>2 /(\alpha+2)$, cf. ibid. p. 243. In particular, $\sum \varrho_{n}<+\infty$. All these sums admit an estimate in terms of $M$ for any $\sigma \in E(M)$. As a matter of example we shall find a bound for the last sum. We have

Theorem 2.10. If $\sigma \in E(M)$ and its Fourier series has the form (2.18), then with the notation (2.10)

$$
\sum_{n=1}^{\infty} \varrho_{n} \leq m(M)+\pi \sqrt{2}(M+\sqrt{M(M+1)}):=\varrho(M) .
$$

Proof. If $\omega(\delta)$ is the modulus of continuity of a continuous, $2 \pi$-periodic function $\sigma$ of total variation $V$ over $[0 ; 2 \pi]$, then the calculations carried out in the proof of Theorem $3.6[6 ;$ p. 241] yield the inequality

$$
\sum_{n=2}^{\infty} \varrho_{n} \leq \frac{1}{2} V^{1 / 2} \sum_{k=1}^{\infty} \omega^{1 / 2}\left(\pi / 2^{k}\right)
$$

In our case $\omega(\delta) \leq 2 \pi(\delta / \pi)^{\alpha}$ and $V \leq 4 \pi$, cf. (1.10) and (2.11). Hence

$$
\sum_{n=2}^{\infty} \varrho_{n} \leq \pi \sqrt{2} \sum_{k=1}^{\infty} 2^{-\alpha k / 2} .
$$

Now, $2^{-\alpha / 2}=(M /(M+1))^{1 / 2}$ and this implies

$$
\sum_{n=2}^{\infty} \varrho_{n} \leq \pi \sqrt{2}(M+\sqrt{M(M+1)}) .
$$

On the other hand

$$
\sigma\left(x-x_{1}\right)=\varrho_{1} \sin x+\varrho_{2} \sin \left(2 x-2 x_{1}+x_{2}\right)+\cdots
$$


and hence

$$
\pi \varrho_{1}=\int_{0}^{2 \pi} \sigma\left(x-x_{1}\right) \sin x d x \leq \int_{0}^{2 \pi}|\sigma(x)| d x \leq \pi m(M)
$$

by (2.14), i.e. $\varrho_{1} \leq m(M)$, hence the inequality (2.19) follows.

Since the Fourier conjugate $\tilde{\sigma}(x)$ of $\sigma$ satisfies $\tilde{\sigma}(x)=\sum_{n=1}^{\infty} \varrho_{n} \cos \left(n x+x_{n}\right)$ we see that $|\tilde{\sigma}(x)| \leq \sum_{n=1}^{\infty} \varrho_{n}$. Thus

$$
\sup \{|\tilde{\sigma}(x)|: x \in \mathbf{R}, \sigma \in E(M)\}:=\tilde{\mu}(M) \leq \varrho(M) .
$$

We shall now prove

Theorem 2.11. Suppose $u\left(e^{i \theta}\right)=\sigma(\theta) \in E(M)$ and $u(z)$ is the harmonic extension of $u\left(e^{i \theta}\right)$ on the unit disk $\mathbf{D}$. Then the Dirichlet integral $D[u]=$ $\iint_{\mathbf{D}}\left(u_{x}^{2}+u_{y}^{2}\right) d x d y$ is finite and

$$
D[u] \leq \pi m(M) \varrho(M)
$$

with $m(M), \varrho(M)$ defined in (2.10) and (2.19), respectively.

Proof. It is well-known that $D[u]=\pi \sum_{n=1}^{\infty} n \varrho_{n}^{2}$, cf. (2.18). In the next section we shall prove that $n \varrho_{n} \leq m(M)$ for all $n \in \mathbf{N}$ and that implies (2.21) in view of (2.19).

We shall now derive another estimate of $\tilde{\sigma}(x)$ in terms of Hölder continuity exponent $\alpha$.

Theorem 2.12. If $\sigma \in E(M)$, then for any $x \in \mathbf{R}$

$$
|\tilde{\sigma}(x)|<4^{1 /(1+\alpha)}\left(1+\alpha^{-1}\right)\left(2^{1-\alpha}-1\right)^{\alpha /(1+\alpha)}:=\tau(M),
$$

where $\alpha$ is given by (1.7).

Proof. Using the well-known formula

$$
\tilde{\sigma}(x)=\frac{1}{\pi} \lim _{\varepsilon \rightarrow 0} \int_{\varepsilon}^{\pi}\left(2 \tan \frac{t}{2}\right)^{-1}(\sigma(x-t)-\sigma(x+t)) d x,
$$

cf. e.g. [6, p. 51], we obtain by means of (1.10) and (2.14):

$$
\begin{aligned}
|\tilde{\sigma}(x)| \leq & \frac{1}{\pi} \int_{0}^{\delta} \frac{t / 2}{\tan (t / 2)}\left(\frac{|\sigma(x-t)-\sigma(x)|}{t^{\alpha}}+\frac{|\sigma(x+t)-\sigma(x)|}{t^{\alpha}}\right) t^{\alpha-1} d t \\
& +\frac{1}{\pi \delta} \frac{\delta / 2}{\tan (\delta / 2)} \int_{-\pi}^{\pi}|\sigma(t)| d t \\
< & \frac{4}{\pi^{\alpha}} \int_{0}^{\delta} t^{\alpha-1} d t+\frac{\pi}{\delta} \frac{M-1}{M+1} \\
= & \frac{4}{\alpha}\left(\frac{\delta}{\pi}\right)^{\alpha}+\frac{\pi}{\delta} \frac{M-1}{M+1}, \quad 0<\delta<\pi .
\end{aligned}
$$


Choosing

$$
\frac{\delta}{\pi}=\left(\frac{1}{4} \frac{M-1}{M+1}\right)^{1 /(1+\alpha)}
$$

we obtain

$$
|\tilde{\sigma}(x)|<4^{1 /(1+\alpha)}\left(1+\alpha^{-1}\right)\left(\frac{M-1}{M+1}\right)^{\alpha /(1+\alpha)} .
$$

Note that, in view of $(1.7),(M-1) /(M+1)=2^{1-\alpha}-1$ and this ends the proof.

It is quite natural to compare the estimates of $\tilde{\mu}(M)$ as given by formulas (2.20) and (2.22). Both expressions $\varrho(M)$ and $\tau(M)$ are $O(M)$ as $M \rightarrow \infty$. More precisely, $\varrho(M) \approx 2 \pi \sqrt{2} M, \tau(M) \approx 4 M \log 2$. However, $\tau(M)=O((M-$ $\left.1)^{1 / 2+o(1)}\right)$, i.e. $\tau(M)=o(1)$, whereas $\varrho(M)=\pi(2+\sqrt{2})+o(1)$ as $M \rightarrow 1$. These facts, as well as computer evaluations, strongly support the presumption that $\tau(M)$ yields a better estimate of $\tilde{\mu}(M)$ for all $M$.

\section{Fourier coefficients of $\sigma$}

We first introduce two classes of analytic functions intimately connected with the class $E(M)$. If $c_{n}=b_{n}+i a_{n}$, then the terms of the series (2.17) have the form $\operatorname{Im} c_{n} e^{i n x}$ which suggests introducing the class $Q(M)$ of functions $q$ analytic in $\mathbf{D}$ and continuous in $\overline{\mathbf{D}}$ :

$$
\left\{q(z)=\sum_{\nu=1}^{\infty} c_{n}(q) z^{n}: \operatorname{Im} q\left(e^{i x}\right)=\sigma(x) \in E(M)\right\}:=Q(M) .
$$

Obviously coefficient problems for $E(M)$ and $Q(M)$ are equivalent. If $q \in Q(M)$, then

$$
F(z)=z \exp q(z)=z+A_{2}(F) z^{2}+\cdots
$$

is evidently a bounded, starlike univalent function. In fact, $F(z) / z$ is analytic, non-vanishing in $\mathbf{D}$, continuous in $\overline{\mathbf{D}}$ and $\arg F\left(e^{i x}\right)=x+\sigma(x)$ increases strictly in $[0 ; 2 \pi)$ by $2 \pi$. We shall denote by $S^{*}(M)$ the corresponding class of functions $F$ which may be called $M$-quasisymmetric starlike functions. Although any $F \in$ $S^{*}(M)$ is associated with an $M$-qs function, it need not have a qc extension, as a simple example $F(z)=z e^{z}$ shows. In this case $F \in S^{*}(M)$ with $M \geq M_{1}=$ $(\pi+2) /(\pi-2)$, cf. Lemma 3.8, however, $F^{\prime}(-1)=0$, i.e. $w=F\left(e^{i t}\right), 0 \leq t \leq 2 \pi$, is not a quasicircle. relation

Since $F \in S^{*}(M)$ implies $\operatorname{Re}\left\{z F^{\prime}(z) / F(z)\right\}>0$, we have the following

$$
q \in Q(M) \quad \Rightarrow \quad 1+z q^{\prime}(z) \in P
$$


where $P$ is the familiar Carathéodory class of normalized functions of positive real part. Thus (3.3) implies in view of (2.18)

$$
n \varrho_{n}(\sigma)=n\left|c_{n}(q)\right| \leq 2 .
$$

The relations (3.3) and (3.4) suggest introducing the constant

$$
\begin{aligned}
c(M) & =\sup \left\{\varrho_{1}(\sigma): \sigma \in E(M)\right\}=\sup \left\{\left|c_{1}(q)\right|: q \in Q(M)\right\} \\
& =\sup \left\{\left|A_{2}(F)\right|: F \in S^{*}(M)\right\} .
\end{aligned}
$$

We shall prove that the constant 2 in (3.4) may be replaced by $c(M)$ which is $<2$ for any $M>1$. In fact, we have, using (2.20)

$$
\left|F\left(e^{i x}\right)\right|=|\exp (i \sigma(x)+\tilde{\sigma}(x))|=\exp \tilde{\sigma}(x) \leq \exp \tilde{\mu}(M)
$$

and hence

$$
c(M)=\sup \left\{\left|A_{2}(F)\right|: F \in S^{*}(M)\right\}<2[1-\exp (-\tilde{\mu}(M))]<2 .
$$

While proving Theorem 2.10 another bound for $c(M)$ has been obtained: $c(M) \leq$ $m(M)$ which is $<2$ for $M<M_{1}$. Thus we have the following:

Theorem 3.1. If $\sigma \in E(M), q \in Q(M)$ have the expansions (2.18) and (3.1), respectively, and are related by (3.1) then

$$
n \varrho_{n}(\sigma)=n\left|c_{n}(q)\right| \leq c(M) \leq \min \{m(M) ; 2[1-\exp (-\tilde{\mu}(M))]\},
$$

where $c(M), \tilde{\mu}(M)$ are defined by (3.5) and (2.20), respectively. The bound $c(M)$ is sharp for all $n \in \mathbf{N}$.

In order to prove this theorem we need some properties of functions $q \in Q(M)$ which are contained in

Lemma 3.2. $Q(M)$ is a convex, compact subset of the space $A(\mathbf{D})$ of functions analytic in D. If $q \in Q(M)$, then also $\overline{q(\bar{z})}, q(a z)$ belong to $Q(M)$ for an arbitrary $a \in \overline{\mathbf{D}}$.

Proof. The first two statements are counterparts of Remark 1.4 and the formula (1.5). If $u(z)=\operatorname{Im} q(z)$, then the condition (1.3), with an arbitrary, fixed real $t$ and $\eta=e^{i t}$, means that the harmonic function $z \mapsto t+u(\eta z)-u(z)$ has the function $M[t+u(z)-u(\bar{\eta} z)]$ (or $M^{-1}[t+u(z)-u(\bar{\eta} z)]$ ) as a harmonic majorant (or minorant). Putting $z=a e^{i x}(|a| \leq 1, x \in \mathbf{R})$ we obtain (1.3) for $\sigma(x)=\operatorname{Im} q\left(a e^{i x}\right)$, i.e. $q(a z) \in Q(M)$.

Corollary 3.3. If $F \in S^{*}(M)$, then $a^{-1} F(a z) \in S^{*}(M)$ for any $a \in \overline{\mathbf{D}}$. 
Proof of Theorem 3.1. Suppose that $q(z)=\sum_{n=1}^{\infty} c_{n} z^{n} \in Q(M)$ and take an arbitrary integer $n \geq 2$. If $\eta=\exp (2 \pi i / n)$, then by Lemma $3.2 q_{k}(z)=$ $q\left(\eta^{k} z\right) \in Q(M)$ for $k=0,1, \ldots, n-1$ and by convexity of $Q(M)$ also $q^{[n]}=$ $n^{-1}\left[q_{0}+q_{1}+\cdots+q_{n-1}\right]$ belongs to $Q(M)$. However, due to obvious equalities

$$
1+\eta^{k}+\eta^{2 k}+\cdots+\eta^{(n-1) k}= \begin{cases}0 & \text { for } 1 \leq k \leq n-1, \\ n & \text { for } k=n,\end{cases}
$$

we have $q^{[n]}(z)=c_{n} z^{n}+c_{2 n} z^{2 n}+\cdots \in Q(M)$. Consider now $q^{*}(z)=c_{n} z+$ $c_{2 n} z^{2}+\cdots$ and put $\sigma^{*}(x)=\operatorname{Im} q^{*}\left(e^{i x}\right)$. Then $\operatorname{Im} q^{[n]}\left(e^{i x}\right)=\sigma^{*}(n x)$. However, $\operatorname{Im} q^{[n]}\left(e^{i x}\right) \in E(M)$ since $q^{[n]} \in Q(M)$, and so $\sigma^{*}(n x) \in E(M)$. Due to the formula (1.2) also $n \sigma^{*}(x) \in E(M)$ which means that $n q^{*} \in Q(M)$. Thus $n c_{n} z+$ $n c_{2 n} z^{2}+\cdots \in Q(M)$ and consequently $n\left|c_{n}(q)\right| \leq c(M)$ for any $n \in \mathbf{N}$. Since the class $Q(M)$ is compact, the bound is sharp for $n=1$. Let $p$ be an extremal function for $n=1$, i.e. $p(z)=c(M) z+\cdots \in Q(M)$ and $\operatorname{Im} p\left(e^{i x}\right)=\sigma_{0}(x) \in$ $E(M)$. The formula (1.2) also shows that $n^{-1} \sigma_{0}(n x) \in E(M)$, i.e. $n^{-1} p\left(z^{n}\right)=$ $n^{-1} c(M) z^{n}+\cdots \in Q(M)$ which implies that the bound $c(M)$ in (3.6) is attained for any $n \in \mathbf{N}$. Since the estimate of $c(M)$ given in (3.6) has been already derived, we are done.

In what follows we shall give estimates of $c(M)$ both from above and below. To this end we first prove a theorem which is of independent interest.

Theorem 3.4. If $\sigma \in E(M)$, then for any $m \in \mathbf{N}$ the $m$ th Fejér mean $\sigma_{m}$ of $\sigma$ also belongs to $E(M)$.

Proof. Let $K_{m}(t)$ denote the Fejér kernel, i.e.

$$
K_{m}(t)=[2 \pi(m+1)]^{-1}\left(\frac{\sin \frac{1}{2}(m+1) t}{\sin \frac{1}{2} t}\right)^{2} .
$$

Then we have for any $m \in \mathbf{N}$

$$
\int_{0}^{2 \pi} K_{m}(u) d u=1, \quad \sigma_{m}(x)=\int_{0}^{2 \pi} \sigma(x+u) K_{m}(u) d u .
$$

Upon putting in (1.3) $x+u$ instead of $x$, multiplying all terms by a non-negative factor $K_{m}(u)$ and integrating over $[0 ; 2 \pi]$ w.r.t. $u$, we obtain using $(3.8)$ the desired inequality (1.3) for $\sigma_{m}$.

Corollary 3.5. If $\sigma \in E(M)$, there exists a sequence of trigonometric polynomials $\left(\sigma_{m}\right)$ of degree $m$ such that $\sigma_{m} \in E(M)$ for any $m \in \mathbf{N}$ and $\lim \sigma_{m}=\sigma$ uniformly on $\mathbf{R}$.

Corollary 3.6. If $\sigma \in E(M)$ has the representation (2.18), then $\sigma_{1}(x)=$ $\frac{1}{2} \varrho_{1} \sin \left(x+x_{1}\right) \in E(M)$. Consequently $\frac{1}{2} c(M) \sin x \in E(M)$. 
Thus we are led to the following problem: Given $M>1$, find the greatest real number $c$ such that the function $x \mapsto c \sin x$ belongs to $E(M)$. To this end we shall prove

Lemma 3.7. Let us define for $t \geq 1$ and $(u, v) \in \mathbf{R}^{2}$ the function (3.9)

$$
F_{t}(u, v)= \begin{cases}(t v+\sin (u+v)-\sin u)) /(t v+\sin u-\sin (u-v)) & \text { for } v \neq 0 \\ 1 & \text { for } v=0\end{cases}
$$

and put

$$
M_{t}=\sup \left\{F_{t}(u, v):(u, v) \in \mathbf{R}^{2}\right\} .
$$

Then $M_{t}$ satisfies

$$
\frac{\pi t+2}{\pi t-2} \leq M_{t} \leq \frac{t+1}{t-1}
$$

or equivalently

$$
\frac{M_{t}-1}{M_{t}+1} \leq \frac{1}{t} \leq \frac{\pi}{2} \frac{M_{t}-1}{M_{t}+1}, \quad t>1 .
$$

Moreover, $M_{t}$ is a continuous, strictly decreasing function of $t>1$ such that

$$
M_{1}=\lim _{t \rightarrow 1+} M_{t}=\frac{\pi+2}{\pi-2}=4.50387 \ldots, \quad \lim _{t \rightarrow \infty} M_{t}=1 .
$$

We have also

$$
\inf \left\{F_{t}(u, v):(u, v) \in \mathbf{R}^{2}\right\}=1 / M_{t}, \quad t>1 .
$$

Proof. Since $F_{t}(u, v)=1 / F_{t}(u,-v),(3.14)$ is evident. Obviously we may restrict ourselves to $v>0$. Note first that the continuity of $M_{t}$ for $t>1$ easily follows from (3.9). We have $M_{t} \geq F_{t}\left(\frac{3}{2} \pi, \pi\right)=(\pi t+2) /(\pi t-2)$, which implies the left hand side inequality in (3.11). We now prove the mononicity statement. Suppose that $1 \leq s<t$ and $M_{t}=F_{t}\left(u_{0}, v_{0}\right)$ with $v_{0}>0$. Hence

$s v_{0}+\sin \left(u_{0}+v_{0}\right)-\sin u_{0}+(t-s) v_{0}=M_{t}\left(s v_{0}+\sin u_{0}-\sin \left(u_{0}-v_{0}\right)\right)+M_{t}(t-s) v_{0}$, i.e.

$$
M_{s} \geq \frac{s v_{0}+\sin \left(u_{0}+v_{0}\right)-\sin u_{0}}{s v_{0}+\sin u_{0}-\sin \left(u_{0}-v_{0}\right)}=M_{t}+\frac{\left(M_{t}-1\right)(t-s) v_{0}}{s v_{0}+\sin u_{0}-\sin \left(u_{0}-v_{0}\right)}
$$


which is greater than $M_{t}$ since the last term is positive. This proves that $M_{t}$ is strictly decreasing.

If $v \neq 0$ and $t>1$, we have by the mean value theorem:

$$
F_{t}(u, v)=\frac{t+\cos \left(u+\vartheta_{1} v\right)}{t+\cos \left(u-\vartheta_{2} v\right)} ; \quad 0<\vartheta_{k}<1, k=1,2,
$$

which implies $(t-1) /(t+1) \leq F_{t}(u, v) \leq(t+1) /(t-1)$ for all $u, v(v \neq 0)$ and $t>1$. This proves the right hand side inequality in (3.11), as well as the second limiting case in (3.13).

We shall be now concerned with the case $t=1$. It is sufficient to consider the range: $0 \leq u \leq 2 \pi, 0 \leq v \leq \pi$, cf. Remark 0.1 .

The partials of $F_{1}$ vanish at points $(u, v)$ which satisfy

$$
(1-\cos v)(v \cos u+\sin v)=0, \quad((1-\cos u)(1-\cos v)-v \sin v)) \sin u=0 .
$$

If $\cos v=1$, or $\sin u=0$, then $F_{1}(u, v)=1$, so we have to assume $v \cos u+\sin v$ $=0, v \sin v=(1-\cos u)(1-\cos v)$, or

$$
\left(1+v^{-1} \sin v\right) \tan (v / 2)-v=0 .
$$

It is easily verified that the left hand side in $(3.15)$ is strictly increasing in $(0 ; \pi)$, so we have to find extremal values of $F_{1}$ on the boundary of the range, i.e. for $v=\pi$. Hence $M_{1}=(\pi+2) /(\pi-2)$ and this proves the first limiting case in (3.13), in view of monotonic behaviour of $M_{t}$.

As an immediate consequence of Lemma 3.7 we obtain

Lemma 3.8. A necessary condition for $c \sin x, c>0$, to belong to some $E(M)$ is that $c \leq 1$. If $M \geq M_{1}$, this condition is also sufficient. If $M \in\left(1 ; M_{1}\right)$ is given, then the condition $c=1 / t_{0}$, where $t_{0}>1$ is the unique solution of the equation $M_{t}=M$, is sufficient for $c \sin x$ to belong to $E(M)$ and $c$ cannot be replaced by any greater number.

Proof. If $c \sin x, c>0$, belongs to some $E(M)$, then $x+c \sin x$ has to be strictly increasing, i.e. $c \leq 1$ is necessary. If $0<c \leq 1$ is given, put $t=c^{-1} \geq 1$. Then the $M$-condition (0.5) for $\sigma(u)=t^{-1} \sin u$ takes the form $M^{-1} \leq F_{t}(u, v)$ $\leq M$ and the sufficiency statement immediately follows from (0.5) and the previous lemma.

Since $\sin x$ belongs to $E(M)$ for $M \geq M_{1}$, we have $c(M) \geq 1$ in this case. Moreover, for $1<M<M_{1}$ we have by means of Corollary $3.61 / t_{0} \leq c(M) \leq 2 / t_{0}$ and this implies, in view of (3.12)

$$
(M-1) /(M+1) \leq c(M) \leq \pi(M-1) /(M+1)=m(M), \quad 1<M<M_{1} .
$$

It is easily seen that (3.16) also holds for $M \geq M_{1}$. 
Corollary 3.9. For any $M>1$ the function $x \mapsto(M-1)(M+1)^{-1} \sin x$ belongs to $E(M)$.

This shows that the norm estimates obtained in Section 2 have a correct order of growth as $M \rightarrow 1$.

A better lower estimate for $c(M)$ can be obtained by considering the function $F(z)=z(1-r z)^{-2}, 0<r<1$. If

$$
g(x)=\arg F\left(e^{i x}\right)=x+\sigma(x),
$$

then

$$
g^{\prime}(x)=1+\sigma^{\prime}(x)=\operatorname{Re} \frac{1+r e^{i x}}{1-r e^{i x}}>0
$$

and hence, by the mean value theorem,

$$
\frac{v+\sigma(u+v)-\sigma(u)}{v+\sigma(u)-\sigma(u-v)} \leq \frac{\max g^{\prime}(x)}{\min g^{\prime}(x)}=\left(\frac{1+r}{1-r}\right)^{2} .
$$

Thus, for $r=(\sqrt{M}-1) /(\sqrt{M}+1), \sigma(x) \in E(M), F(z) \in S^{*}(M)$ and by (3.5)

$$
A_{2}(F)=2 r=\frac{M-1}{(M+1)\left(\frac{1}{2}+\sqrt{M} /(1+M)\right)} \leq c(M),
$$

which improves the lower estimate in (3.16) and is valid for all $M>1$. The estimates (3.17) and (3.4) imply $\lim _{M \rightarrow \infty} c(M)=2$.

It seems that the final solution of the Fourier coefficient problem for the class $E(M)$, i.e. the determination of the constant $c(M)$, could be achieved by settling the problem of the second coefficient in the class of $M$-quasisymmetric starlike functions. 


\section{References}

[1] Ahlfors, L.V.: Lectures on quasiconformal mappings. - Van Nostrand, Princeton-Toronto -New York-London, 1966.

[2] Beurling, A., and L.V. AhlFors: The boundary correspondence under quasiconformal mappings. - Acta Math. 96, 1956, 125-142.

[3] Dienes, P.: The Taylor series. - Oxford University Press, Oxford, 1931.

[4] Kelingos, J.A.: Boundary correspondence under quasiconformal mappings. - Michigan Math. J. 13, 1966, 235-249.

[5] Krzyż, J.G.: Quasicircles and harmonic measure. - Ann. Acad. Sci. Fenn. Ser. A I Math. 12, 1987, 19-24.

[6] Zygmund, A.: Trigonometric series, Vol. 1. - Cambridge University Press, Cambridge, 1968.

Maria Curie-Sklodowska University

Institute of Mathematics

Pl. M. Curie-Sklodowskiej 1

PL-20031 Lublin

Poland

Received 15 October 1987 